\title{
QUANTITATIVE STABILITY IN THE ISODIAMETRIC INEQUALITY VIA THE ISOPERIMETRIC INEQUALITY
}

\author{
FRANCESCO MAGGI, MARCELLO PONSIGLIONE, AND ALDO PRATELLI
}

\begin{abstract}
The isodiametric inequality is derived from the isoperimetric inequality through a variational principle, establishing that balls maximize the perimeter among convex sets with fixed diameter. This principle also brings quantitative improvements to the isodiametric inequality, shown to be sharp by explicit nearly optimal sets.
\end{abstract}

\section{INTRODUCTION}

The isodiametric inequality is the analytical formulation of the basic variational principle that balls maximize volume under a diameter constraint. If $B_{1}$ denotes the unit ball in the Euclidean space $\mathbb{R}^{n}, n \geq 1$, and $|E|$ is the Lebesgue measure of a bounded measurable set $E \subset \mathbb{R}^{n}$ with diameter $\operatorname{diam}(E)$, then the isodiametric inequality takes the form

$$
\left(\frac{\operatorname{diam}(E)}{2}\right)^{n}\left|B_{1}\right| \geq|E|,
$$

where equality holds if and only if $E$ is (equivalent to) a ball. The aim of this paper is to provide some sharp and natural results about the stability of balls as maximizers in the isodiametric variational problem. This amounts to estimating suitable notions of distance of $E$ from the family of balls in terms of its isodiametric deficit,

$$
\delta(E)=\left(\frac{\operatorname{diam}(E)}{2}\right)^{n} \frac{\left|B_{1}\right|}{|E|}-1 .
$$

The isodiametric deficit $\delta$ is invariant by scaling, rigid motions, and it is nonnegative, with $\delta(E)=0$ if and only if $E$ is equivalent to a ball.

The stability results. We now state our stability results. We shall assume (without loss of generality) that $\operatorname{diam}(E)=2$, and we shall directly focus on the case $n \geq 2$ to avoid trivialities. Our first result concerns a quantitative improvement of (1.1) involving the $L^{1}$-distance of $E$ from the family of balls. We set the following notation:

$$
B_{r}(x)=\left\{y \in \mathbb{R}^{n}:|y-x|<r\right\}, \quad B_{r}=B_{r}(0) .
$$

Received by the editors April 26, 2011.

2010 Mathematics Subject Classification. Primary 51N20.

Key words and phrases. Isodiametric inequality, geometric inequalities.

(C) 2013 American Mathematical Society Reverts to public domain 28 years from publication 
Theorem 1. If $E \subset \mathbb{R}^{n}$ is a measurable set with $\operatorname{diam}(E)=2$, then there exists $x \in \mathbb{R}^{n}$ such that

$$
C(n) \delta(E)^{1 / 2} \geq \frac{\left|E \Delta B_{1}(x)\right|}{\left|B_{1}\right|},
$$

where $C(n)$ is a constant depending only on $n$.

Notice that (1.2) is equivalent to

$$
\left|B_{1}\right| \geq|E|\left\{1+\frac{1}{C(n)^{2}}\left(\frac{\left|E \Delta B_{1}(x)\right|}{\left|B_{1}\right|}\right)^{2}\right\} .
$$

Moreover, a possible value for the constant $C(n)$ in (1.2) and (1.3) is the following (where $\left.n^{\prime}:=n /(n-1)\right)$ :

$$
C(n)=\frac{181 n^{3}}{\left(2-2^{1 / n^{\prime}}\right)^{3 / 2}}+1 .
$$

Next, we look for uniform bounds on the distance between $E$ and a ball of equal diameter. More precisely, given a set $E$ with $\operatorname{diam}(E)=2$, we shall introduce the radii

$$
\begin{aligned}
r_{E}^{\text {out }} & =\inf _{x \in \mathbb{R}^{n}} \inf \left\{r>0: E \subset B_{1+r}(x)\right\}, \\
r_{E}^{\text {in }} & =\inf _{x \in \mathbb{R}^{n}} \inf \left\{r>0: B_{1}(x) \subset E+B_{r}\right\} .
\end{aligned}
$$

Of course, one has that $r_{E}^{\text {out }}=0$ and $r_{E}^{\text {in }}=0$ if and only if $E$ is the unit ball, hence, if and only if $\delta(E)=0$. Our second stability estimate shows that $r_{E}^{\text {out }}$ and $r_{E}^{\text {in }}$ can be bounded from above by suitable powers of $\delta(E)$.

Theorem 2. Let $E$ be a measurable subset of $\mathbb{R}^{n}$ with $\operatorname{diam}(E)=2$. There exist two positive constants $K_{\text {in }}(n)$ and $K_{\text {out }}(n)$ such that

$$
r_{E}^{\text {out }} \leq \begin{cases}K_{\text {out }}(2) \delta(E)^{1 / 2} & \text { if } n=2, \\ K_{\text {out }}(3)(\delta(E) \max \{|\log \delta(E)|, 1\})^{1 / 2} & \text { if } n=3, \\ K_{\text {out }}(n) \delta(E)^{2 /(n+1)} & \text { if } n>3\end{cases}
$$

and

$$
r_{E}^{i n} \leq K_{\text {in }}(n) \delta(E)^{1 / n} .
$$

Remark 3. From Theorem 2 we easily deduce a quantitative estimate for the Hausdorff distance between $E$ and the family of balls. More precisely, let $d_{H}$ denote the Hausdorff distance between compact sets on $\mathbb{R}^{n}$, and notice that by immediate geometric arguments one has

$$
\max \left\{r_{E}^{\text {in }}, r_{E}^{\text {out }}\right\} \leq \alpha(E):=\inf _{x \in \mathbb{R}^{n}} d_{H}\left(E, B_{1}(x)\right) \leq 2 \max \left\{r_{E}^{\text {in }}, r_{E}^{\text {out }}\right\} .
$$

Hence, under the same assumption as in Theorem 2, we have

$$
\alpha(E) \leq 2 \max \left\{r_{E}^{\text {in }}, r_{E}^{\text {out }}\right\} \leq 2 K(n) \delta(E)^{1 / n} .
$$

Of course, this estimate is weaker than Theorem 2. as it hides the fact that a stronger estimate holds true for $r_{E}^{\text {out }}$. 
Strategy of the proof. In recent years, several stability estimates have been proved for various geometric inequalities, involving perimeters, capacities, eigenvalues and other relevant set functionals. Usually, the starting point of these results is the choice of an argument characterizing the optimal sets in the variational problem under consideration. In the case of the isodiametric inequality, our choice could have been the well-known argument by Bieberbach Bi based on Steiner symmetrization (see [EG, Section 2.2]). However, due to the elusive nature of the diameter constraint, it is unclear how to "perturb" Bieberbach's proof in order to obtain sharp quantitative stability estimates. We have avoided such difficulties thanks to a fruitful link between the isodiametric and the isoperimetric problem (Theorem 5 and Remark 7). In Section 2 we shall exploit this connection in order to derive the above stability estimates for the isodiametric inequality from the analogous stability estimates for the isoperimetric inequality. The sharpness of the above theorems shall then be discussed in Section 3. through the construction of suitable families of nearly optimal sets, obtained by perturbing the unit ball. To show the optimality of (1.5) it is enough to remove a small ball of radius $\delta^{1 / n}$ inside $B_{1}$, while nearly optimal sets for (1.2) and (1.4) are obtained, loosely speaking, by adding a sickle shaped set around a point $x \in \partial B_{1}$, while removing from $B_{1}$, around the antipodal point $-x$, the minimal amount of mass needed in order to keep the diameter constraint. The size of the optimal perturbation for (1.4) turns out to depend on the dimension: it is very concentrated around $x$ in the case $n>3$, while it is diffuse for $n=2$. The case $n=3$ is somehow critical, and requires a specific construction of the optimal sickle shaped perturbation.

Remark 4 (Explicit constants). We stress the fact that, as we are going to discuss later on, the dimensional constants appearing in the above estimates are explicitly computable. This is, of course, stronger information than the existence of a constant depending on the dimension $n$ only. The problem of determining the optimal constants in these estimates seems particularly difficult. In the case of the isoperimetric inequality, for example, this kind of question has been settled only in the planar case $n=2$ Ca, AFN, CL2. We may also notice that, by (2.5), the optimal constants $C(n)$ in (1.2) are smaller than the optimal constants $C_{0}(n)$ in (2.2).

Connection between the isodiametric and the isoperimetric problem. Let us recall that, whenever $F \subset \mathbb{R}^{n}$ is a Lebesgue measurable set with $|F|<\infty$, the (Euclidean) isoperimetric inequality states that

$$
P(F) \geq n\left|B_{1}\right|^{1 / n}|F|^{(n-1) / n},
$$

with equality if and only if $F$ is equivalent to a ball. Here, $P(F)$ denotes the distributional perimeter of $F$, a quantity that agrees with $\mathcal{H}^{n-1}(\partial F)$ whenever $F$ is either an open set with $C^{1}$-boundary, a convex set, or a polyhedron. Now let $E$ be a set in $\mathbb{R}^{n}$ with $\operatorname{diam}(E)=2$, and let $F$ be the convex envelope of $E$. The bridge between the isodiametric inequality and the isoperimetric inequality is provided by the following variational principle: among convex sets with fixed diameter, balls maximize perimeter.

Theorem 5. If $F$ is a convex set in $\mathbb{R}^{n}$ with $\operatorname{diam}(F)=2$, then

$$
P(F) \leq P\left(B_{1}\right) .
$$

When $n \geq 3$, equality holds in (1.8) if and only if $F$ is equivalent to a ball. 

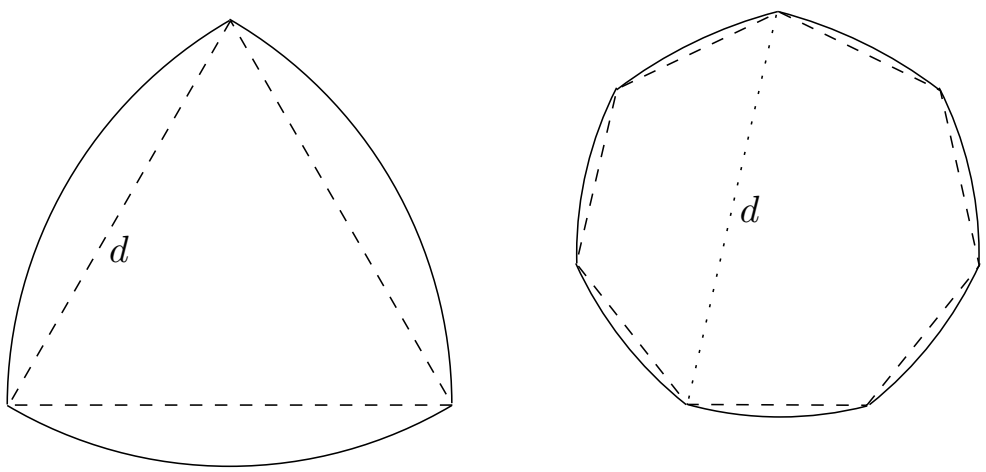

Figure 1. The regular Reuleaux polygons of diameter $d$ have perimeter $\pi d$, and are thus optimal in (1.8). Twenty and fifty British pence are regular Reuleaux eptagons [Mo, Section 3].

Remark 6 (Reuleaux polygons). In the case $n=2$, balls do not exhaust the equality cases in (1.8). Indeed, it turns out that every Reuleaux polygon of diameter 2 satisfies equality in (1.8). A nice and complete introduction to these shapes is found, for example, in [Mo, Section 3]. For example, let us recall that a regular Reuleaux polygon is a convex set which is obtained by starting from a regular polygon with an odd number of sides and replacing the edges with circular arcs: each arc is centered in a given vertex and passes through the two vertices of the opposite edge (the regular Reuleaux triangle and eptagon are represented in Figure10. In general, every Reuleaux polygon of diameter $d$ has perimeter $\pi d$; moreover, every bounded convex polygon is contained in a Reuleaux polygon of the same diameter. These two properties lead immediately to prove Theorem 5 in the planar case $n=2$.

Remark 7 (The isodiametric principle from the isoperimetric principle). Let us prove the isodiametric inequality (1.1), combining Theorem 5 with the isoperimetric inequality. Given $E \subset \mathbb{R}^{n}$, with $\operatorname{diam}(E)=2$, we want to prove that $|E| \leq\left|B_{1}\right|$. Indeed, applying the isoperimetric inequality to the convex hull $F$ of $E$, which clearly has the same diameter of $E$, and taking Theorem 5 into account, we find that

$$
n\left|B_{1}\right|^{1 / n}|E|^{(n-1) / n} \leq n\left|B_{1}\right|^{1 / n}|F|^{(n-1) / n} \leq P(F) \leq P\left(B_{1}\right)=n\left|B_{1}\right|,
$$

that is, $|E| \leq\left|B_{1}\right|$. If $|E|=\left|B_{1}\right|$, then by (1.9) we get $|F|=|E|$ and $P(F)=P\left(B_{1}\right)$. In particular $E$ is equivalent to its convex envelope $F$, which in turn is optimal in the isoperimetric inequality, and thus is equivalent to a unit ball. In conclusion, $E$ is equivalent to a unit ball. Clearly, these arguments can be exploited in order to bound the isoperimetric deficit $\delta^{\prime}(F)$ of $F$ in terms of the isodiametric deficit $\delta(E)$ of $E$; see Lemma 8 below.

Proof of Theorem 5. We argue by induction over the dimension $n$, the case $n=1$ being trivial. For every $\nu \in \partial B_{1}$, let $F_{\nu}$ be the projection of $F$ over the orthogonal space $\nu^{\perp}$ to $\nu$. Let $B_{1}^{n-1}$ be the Euclidean unit ball in $\mathbb{R}^{n-1}$, and set $\omega_{n-1}=$ $\mathcal{H}^{n-1}\left(B_{1}^{n-1}\right)$. By the Cauchy Formula, we have

$$
P(F)=\int_{\partial B_{1}} \frac{\mathcal{H}^{n-1}\left(F_{\nu}\right)}{\omega_{n-1}} d \mathcal{H}^{n-1}(\nu) .
$$


Since $\operatorname{diam}\left(F_{\nu}\right) \leq 2$, the isodiametric inequality in $\mathbb{R}^{n-1}$ implies $\mathcal{H}^{n-1}\left(F_{\nu}\right) \leq \omega_{n-1}$, and thus $P(F) \leq P\left(B_{1}\right)$. The discussion of equality cases for $n \geq 3$ is then achieved by a powerful result of Howard $[\mathrm{Ho}$. Indeed, let us now assume that $n \geq 3$ and $P(F)=P\left(B_{1}\right)$. By the above argument, we have $\mathcal{H}^{n-1}\left(F_{\nu}\right)=\omega_{n-1}$ for a.e. $\nu \in \partial B_{1}$, i.e., $F_{\nu}$ is optimal in the isodiametric inequality in $\mathbb{R}^{n-1}$, and thus it is an $(n-1)$-dimensional unit disk in $\nu^{\perp}$. In particular, $F$ is a convex set with constant width and brightness: by Howard's Theorem [Ho, Corollary 2], $F$ is a ball.

\section{Proof of the Stability estimates}

Given a Lebesgue measurable set $F \subset \mathbb{R}^{n}$, we introduce the isoperimetric deficit $\delta^{\prime}(F)$ of $F$, defined as

$$
\delta^{\prime}(F)=\frac{P(F)}{n\left|B_{1}\right|^{1 / n}|F|^{(n-1) / n}}-1 .
$$

Like the isodiametric deficit, the isoperimetric deficit is invariant by scaling and by rigid motions. The isoperimetric inequality (1.7) amounts to saying that $\delta^{\prime}(F) \geq 0$, with $\delta^{\prime}(F)=0$ if and only if $F$ is equivalent to a ball. The starting point of our analysis is the following elementary lemma, relating the isodiametric deficit of a bounded set to the isoperimetric deficit of its convex envelope.

Lemma 8. If $E \subset \mathbb{R}^{n}$ and $F$ is the convex envelope of $E$, then

$$
\delta^{\prime}(F) \leq \delta(E) .
$$

Proof. Since the isodiametric deficit and the isoperimetric deficit are invariant by scaling, without loss of generality we may assume that $2=\operatorname{diam}(E)=\operatorname{diam}(F)$. By Theorem 5 and since $|F| \leq\left|B_{1}\right|$ by the isodiametric inequality, we obtain

$$
\begin{aligned}
\delta^{\prime}(F) & =\frac{P(F)}{n\left|B_{1}\right|^{1 / n}|F|^{(n-1) / n}}-1 \leq \frac{P\left(B_{1}\right)}{n\left|B_{1}\right|^{1 / n}|F|^{(n-1) / n}}-1=\frac{n\left|B_{1}\right|}{n\left|B_{1}\right|^{1 / n}|F|^{(n-1) / n}}-1 \\
& =\left(\frac{\left|B_{1}\right|}{|F|}\right)^{\frac{n-1}{n}}-1 \leq \frac{\left|B_{1}\right|}{|E|}-1=\delta(E),
\end{aligned}
$$

that is, (2.1).

Starting from Lemma 8 , Theorem 1 is now a corollary of the following theorem, first proved in [FMP] (for alternative approaches see [FiMP, CL1]).

Theorem ([FMP $)$. If $F$ is a measurable set with $|F|<\infty$, then there exists $x \in \mathbb{R}^{n}$ such that

$$
C_{0}(n) \sqrt{\delta^{\prime}(F)} \geq \frac{\left|F \Delta\left(x+t_{F} B_{1}\right)\right|}{|F|},
$$

where $t_{F}=\left(|F| /\left|B_{1}\right|\right)^{1 / n}$ and $C_{0}(n)$ is a constant depending on the dimension $n$ only.

It is important to recall that the mass transportation approach developed in FiMP allows us to derive (2.2) with an explicit value for $C_{0}(n)$. Namely, setting $n^{\prime}=n /(n-1)$, one can take

$$
C_{0}(n)=\frac{181 n^{3}}{\left(2-2^{1 / n^{\prime}}\right)^{3 / 2}} .
$$

We are now in a position to prove Theorem 1 
Proof of Theorem 1. Let $F$ be the convex envelope of $E$. By Lemma 8 and by the quantitative isoperimetric inequality (2.2), since $|F| \leq\left|B_{1}\right|$ we find that, up to a translation,

$$
C_{0}(n) \sqrt{\delta(E)} \geq C_{0}(n) \sqrt{\delta^{\prime}(F)} \geq \frac{\left|F \Delta\left(t_{F} B_{1}\right)\right|}{|F|} \geq \frac{\left|F \Delta\left(t_{F} B_{1}\right)\right|}{\left|B_{1}\right|} .
$$

By the triangular inequality we have

$$
\left|F \Delta B_{1}\right| \leq\left|F \Delta\left(t_{F} B_{1}\right)\right|+\left|\left(t_{F} B_{1}\right) \Delta B_{1}\right|=\left|F \Delta\left(t_{F} B_{1}\right)\right|+\left(\left|B_{1}\right|-|F|\right) .
$$

From (2.3) and (2.4) we get

$$
\begin{aligned}
\left|B_{1} \Delta E\right| & \leq\left|B_{1} \Delta F\right|+|F \Delta E| \leq\left|F \Delta\left(t_{F} B_{1}\right)\right|+\left(\left|B_{1}\right|-|F|\right)+(|F|-|E|) \\
& \leq C_{0}(n)\left|B_{1}\right| \sqrt{\delta(E)}+\left(\left|B_{1}\right|-|E|\right) \leq\left|B_{1}\right|\left(C_{0}(n) \sqrt{\delta(E)}+\delta(E)\right),
\end{aligned}
$$

from which we immediately achieve the proof of (1.2) with $C(n)=C_{0}(n)+1$ under the assumption that $\delta(E) \leq 1$. If, otherwise, $\delta(E) \geq 1$, then

$$
\frac{\left|B_{1} \Delta E\right|}{\left|B_{1}\right|} \leq \frac{\left|B_{1}\right|+|E|}{\left|B_{1}\right|} \leq 2 \leq 2 \sqrt{\delta(E)}
$$

and (1.2) follows as $C_{0}(n) \geq 1$.

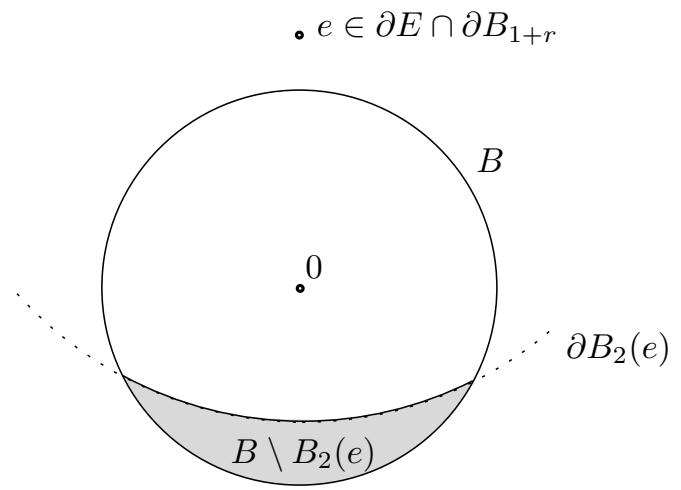

Figure 2. The measure $\left|B_{1} \backslash B_{2}(e)\right|$ behaves like $\left(r_{E}^{\text {out }}\right)^{(n+1) / 2}$.

Remark 9. A non-sharp form of Theorem 2 easily follows from Theorem 1 Indeed, up to a translation we can always assume that (1.2) holds true with $x=0$. With this assumption, an immediate geometric argument shows that, calling $\bar{x}$ the point such that $E \subset B_{1+r_{E}^{\text {out }}}(\bar{x})$, one has $|\bar{x}| \leq C^{\prime}(n) r_{E}^{\text {out }}$, with $C^{\prime}(n)$ being a constant only depending on $n$. As a consequence, by definition of $r_{E}^{\text {out }}$ there exists some point

$$
e \in \partial E \cap \partial B_{1+r}
$$

for some $r_{E}^{\text {out }} \leq r \leq\left(1+C^{\prime}(n)\right) r_{E}^{\text {out }}$.

Since $\operatorname{diam}(E)=2$, it must be $E \subset \bar{B}_{2}(e)$. In particular, from (1.2) (with $x=0$ ) we derive the lower bound

$$
\left|B_{1}\right| C(n) \sqrt{\delta(E)} \geq\left|B_{1} \backslash E\right| \geq\left|B_{1} \backslash B_{2}(e)\right| .
$$


If $r_{E}^{\text {out }}$, and hence $r$, is small, then the set $B_{1} \backslash B_{2}(e)$ is, roughly speaking, a thin set with "height" of order $r$ and "cross section" of order $r^{1 / 2}$; see Figure 2, Therefore,

$$
\left|B_{1} \backslash B_{2}(e)\right| \approx r \cdot r^{(n-1) / 2} \approx\left(r_{E}^{\text {out }}\right)^{(n+1) / 2} .
$$

Thus, this simple argument suffices to prove the stability estimate

$$
r_{E}^{\text {out }} \leq C(n) \delta(E)^{1 /(n+1)},
$$

which however provides us with a non-sharp decay rate for $r_{E}^{\text {out }}$ in terms of $\delta(E)$.

We now turn to the proof of Theorem 2. In this case, we are going to apply a beautiful stability result for the isoperimetric inequality which is due to Bernstein $\mathrm{Be}$ and Bonnesen $\mathrm{BO}$ in the planar case $n=2$, and to Fuglede $\mathrm{Fu}$ in higher dimensions, where a bound for $\alpha$, as defined in (1.6), is provided in terms of the isoperimetric deficit $\delta^{\prime}$. We remark that the constants $K(n)$ appearing in the statement are explicitly computable from Fuglede's work [Fu], and that all the exponents are sharp.

Theorem ( $\mathrm{Be}, \mathrm{Bo}, \mathrm{Fu})$. There exist two positive constants $\eta_{0}(n)$ and $K_{0}(n)$ with the following property. If $G \subset \mathbb{R}^{n}$ is a convex set with $|G|=\left|B_{1}\right|$ and $\delta^{\prime}(G) \leq \eta_{0}(n)$, then

$$
\alpha(G) \leq \begin{cases}K_{0}(2) \delta^{\prime}(G)^{1 / 2} & \text { if } n=2, \\ K_{0}(3)\left(\delta^{\prime}(G)\left|\log \delta^{\prime}(G)\right|\right)^{1 / 2} & \text { if } n=3, \\ K_{0}(n) \delta^{\prime}(G)^{2 /(n+1)} & \text { if } n \geq 4 .\end{cases}
$$

Proof of Theorem 2. Since $\operatorname{diam}(E)=2$, we have $r_{E}^{\text {out }} \leq 1$ and $r_{E}^{\text {in }} \leq 1$. For every $n \geq 2$, let us consider the function $\varphi_{n}:(0, \infty) \rightarrow(0, \infty)$ defined as

$$
\varphi_{n}(\delta)=\left\{\begin{array}{ll}
\delta^{1 / 2}, & \text { if } n=2, \\
(\delta \max \{|\log \delta|, 1\})^{1 / 2}, & \text { if } n=3, \\
\delta^{2 /(n+1)}, & \text { if } n \geq 4,
\end{array} \quad \delta>0 .\right.
$$

It is immediate to notice that the function $\varphi$ is increasing for any $n$. Let us also set $\eta(n)=\min \left\{\eta_{0}(n), e^{-1}\right\}$, so that by Fuglede's Theorem one has that

$$
\alpha(G) \leq K_{0}(n) \varphi_{n}\left(\delta^{\prime}(G)\right) \quad \forall G \subset \mathbb{R}^{n}, G \text { convex, } 0 \leq \delta(G) \leq \eta(n) .
$$

Estimate for $r_{E}^{\text {out }}$ : Let $E \subset \mathbb{R}^{n}$ be a set with $\operatorname{diam}(E)=2$. If $\delta(E) \geq \eta(n)$, then, since $\varphi$ is increasing, we clearly have

$$
r_{E}^{\text {out }} \leq 1 \leq \frac{\varphi_{n}(\delta(E))}{\varphi_{n}(\eta(n))} .
$$

Otherwise, assuming that $\delta(E)<\eta(n)$, let $F$ be the convex envelope of $E$, and let $G=\left(\left|B_{1}\right| /|F|\right)^{1 / n} F$. By convexity, $r_{E}^{\text {out }}=r_{F}^{\text {out }}$. By the isodiametric inequality $|F| \leq\left|B_{1}\right|$, we thus have $r_{G}^{\text {out }} \geq r_{F}^{\text {out }}=r_{E}^{\text {out }}$. By scale invariance of the deficit and by Lemma 8

$$
\delta^{\prime}(G)=\delta^{\prime}(F) \leq \delta(E) \leq \eta(n) .
$$

Therefore, also taking into account (1.6), (2.6) and the monotonicity of $\varphi$, we have

$$
r_{E}^{\text {out }} \leq r_{G}^{\text {out }} \leq \alpha(G) \leq K_{0}(n) \varphi_{n}\left(\delta^{\prime}(G)\right) \leq K_{0}(n) \varphi_{n}(\delta(E)) .
$$

In conclusion, the estimate (1.4) holds true with the choice

$$
K_{\text {out }}(n)=\max \left\{K_{0}(n), \frac{1}{\varphi_{n}\left(\eta_{n}\right)}\right\} \text {. }
$$




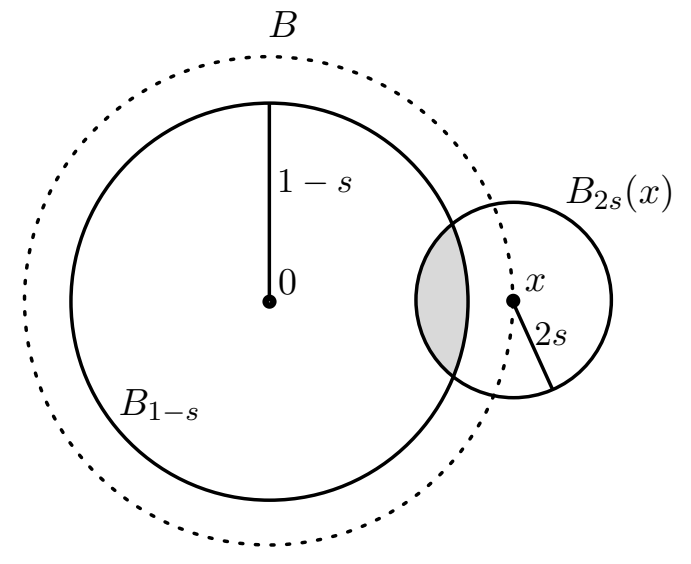

Figure 3. The worst possible situation in (2.16).

Estimate for $r_{E}^{i n}$ : Exactly as in (2.7), if $\delta(E) \geq \eta(n)$, we know

$$
r_{E}^{\text {in }} \leq 1 \leq \frac{\delta(E)^{1 / n}}{\eta(n)^{1 / n}} .
$$

On the other hand, if $\delta(E) \leq \eta(n)$, then (2.8) is in force. As a consequence, if $r_{E}^{\text {in }} \leq 2 r_{E}^{\text {out }}$, then we immediately get

$$
r_{E}^{\text {in }} \leq 2 r_{E}^{\text {out }} \leq 2 K_{0}(n) \varphi_{n}(\delta(E)) \leq 6 e^{-1} K_{0}(n) \delta(E)^{1 / n}
$$

using the trivial fact that $\varphi_{n}(t) \leq 3 e^{-1} t^{1 / n}$ for all $n$ and for all $0 \leq t \leq \eta(n)$.

We are then left to consider the last possible case, namely, if

$$
\delta(E) \leq \eta(n), \quad r_{E}^{\text {in }}>2 r_{E}^{\text {out }} .
$$

By definition of $r_{E}^{\text {out }}$ we know that, up to a translation,

$$
E \subset B_{1+r_{E}^{\text {out }}} .
$$

By the definition of $r_{E}^{\text {in }}$, it readily follows the existence of $x \in B_{1}$ such that

$$
B_{r_{E}^{\text {in }}}(x) \subset \mathbb{R}^{n} \backslash E .
$$

Let us set $H=E \cup B_{1-r_{E}^{\text {out }}}$. By (2.12), we find $\operatorname{diam}(H)=\operatorname{diam}(E)=2$. By the isodiametric inequality, we find $0 \leq\left|B_{1}\right|-|H|=\left|B_{1} \backslash H\right|-\left|H \backslash B_{1}\right|$, which implies

$$
\left|\left(B_{1} \backslash B_{1-r_{E}^{\text {out }}}\right) \backslash E\right|=\left|B_{1} \backslash H\right| \geq\left|H \backslash B_{1}\right|=\left|E \backslash B_{1}\right| .
$$

Therefore,

$$
\begin{aligned}
\left|B_{1}\right| \delta(E) & \geq|E| \delta(E)=\left|B_{1}\right|-|E|=\left|B_{1} \backslash E\right|-\left|E \backslash B_{1}\right| \\
& =\left|B_{1-r_{E}^{\text {out }}} \backslash E\right|+\left|\left(B_{1} \backslash B_{1-r_{E}^{\text {out }}}\right) \backslash E\right|-\left|E \backslash B_{1}\right| \\
\text { by (2.14) } & \geq\left|B_{1-r_{E}^{\text {out }}} \backslash E\right| \\
\text { by (2.13) } & \geq\left|B_{1-r_{E}^{\text {out }}} \cap B_{r_{E}^{\text {in }}}(x)\right| \\
\text { by (2.11) } & \geq\left|B_{1-\left(r_{E}^{\text {in }} / 2\right)} \cap B_{r_{E}^{\text {in }}}(x)\right| .
\end{aligned}
$$


Notice now that, for every $s \in(0,1 / 2)$ and every $x \in \overline{B_{1}}$, one has (see Figure 3 . which shows the "worst" case, namely when $x \in \partial B_{1}$ )

$$
B_{s / 2}\left(\left(1-\frac{3}{2} s\right) x\right) \subset B_{1-s} \cap B_{2 s}(x) .
$$

As a consequence, recalling that $r_{E}^{\text {in }} \leq 1$, from (2.15) we deduce

$$
\delta(E) \geq\left(\frac{r_{E}^{\text {in }}}{4}\right)^{n},
$$

that is,

$$
r_{E}^{\text {in }} \leq 4 \delta(E)^{1 / n} .
$$

Finally, putting together (2.9), (2.10) and (2.17), we obtain the validity of (1.5) as soon as we take

$$
K_{\text {in }}(n) \geq \max \left\{\frac{1}{\eta(n)^{1 / n}}, 6 e^{-1} K_{0}(n), 4\right\} .
$$

We have then concluded the proof of Theorem 2 .

\section{NeARLY OPTIMAL SETS}

This section is devoted to the construction of examples showing the optimality of the decay rates provided in Theorem 1 and Theorem 2 These examples play around the notions of axially symmetric sets and of rearrangement by spherical caps, that are briefly reviewed in Section 3.1. The examples are then constructed in Section 3.2. Before coming to this, we settle the proof of the optimality of (1.5).

Example 10 (Optimality of (1.5)). It is enough to pick up $x \in B_{1}$, and set $E=B_{1} \backslash B_{r}(x)$ for some $r \in\left(0, \operatorname{dist}\left(x, \partial B_{1}\right)\right)$. Then we have

$$
\delta(E)=\frac{\left|B_{r}(x)\right|}{|E|}=\frac{r^{n}}{1-r^{n}}, \quad r_{E}^{\text {in }}=r .
$$

The decay rate found in estimate (1.5) is thus trivially optimal for any dimension $n$.

3.1. Rearrangement by spherical caps. We briefly discuss the properties of the rearrangement by spherical caps in connection with our problem. We introduce some notation for sets enjoying such symmetry, that will be used in the construction of our nearly optimal sets. Finally, we prove that rearrangement by spherical caps transforms a bounded measurable set $E$ into an axially symmetric set $E_{s c}$ with the same measure and possibly lower diameter. This result is not strictly needed in our constructions, but gives a flavor of why we are looking for nearly optimal sets in this class.

Consider the geodesic distance $d$ on $\partial B_{1}$. For every $A \subset \partial B_{1}$ and $\alpha \in(0, \pi)$, let $I_{\alpha} A$ be the $\alpha$-neighborhood of $A$ in $\partial B_{1}$ (with respect to the geodesic distance $d$ ), defined by

$$
I_{\alpha} A=\left\{q \in \partial B_{1}: d(q, A)<\alpha\right\} .
$$

Moreover, given $e \in \partial B_{1}$ and $\alpha \in[0, \pi]$, let us denote by $K[e, \alpha]$ the spherical cap contained in $\partial B_{1}$ with center at $e$ and geodetic radius $\alpha$. In other words,

$$
K[e, \alpha]:=\left\{q \in \partial B_{1}: d(e, q)<\alpha\right\}=I_{\alpha}\{e\} .
$$


Then the following Brunn-Minkowski type inequality on $\partial B_{1}$ holds true (see, e.g., Ga, Section 12]):

$$
\mathcal{H}^{n-1}\left(I_{\alpha} A\right) \geq \mathcal{H}^{n-1}\left(I_{\alpha} K[e, \beta]\right),
$$

whenever $\beta$ is such that $\mathcal{H}^{n-1}(A)=\mathcal{H}^{n-1}(K[e, \beta])$.

Now let $E$ be a bounded measurable subset of $\mathbb{R}^{n}$. We associate to $E$ the measurable function $v_{E}:(0, \infty) \mapsto[0, \pi]$, defined so that

$$
r^{n-1} \mathcal{H}^{n-1}\left(K\left[e, v_{E}(r)\right]\right)=\mathcal{H}^{n-1}\left(E \cap \partial B_{r}\right), \quad r>0,
$$

where $B_{r}$ denotes the ball or radius $r$ centered at zero. The rearrangement by spherical caps $E_{s c}$ of $E$, with center at the origin and axis $e \in \partial B_{1}$, is thus defined as

$$
E_{s c}=\left\{p \in \mathbb{R}^{n}: p \in|p| K\left[e, v_{E}(|p|)\right]\right\} .
$$

In the next theorem we prove that, as in the case of Steiner symmetrization, the diameter decreases under symmetrization by spherical caps.

Theorem 11. For every bounded measurable set $E$ we have $\left|E_{s c}\right|=|E|$ and

$$
\operatorname{diam}\left(E_{s c}\right) \leq \operatorname{diam}(E) .
$$

Proof. The identity $\left|E_{s c}\right|=|E|$ being elementary, we directly focus on the inequality $\operatorname{diam}\left(E_{s c}\right) \leq \operatorname{diam}(E)$. To this purpose, let $p, q \in \partial B_{1}$ and $r_{p}, r_{q} \in \mathbb{R}$ be such that $r_{p} p$ and $r_{q} q$ belong to $\bar{E}_{s c}$, and $\left|r_{p} p-r_{q} q\right|=\operatorname{diam}\left(E_{s c}\right)$. Since $r_{p} p$ and $r_{q} q$

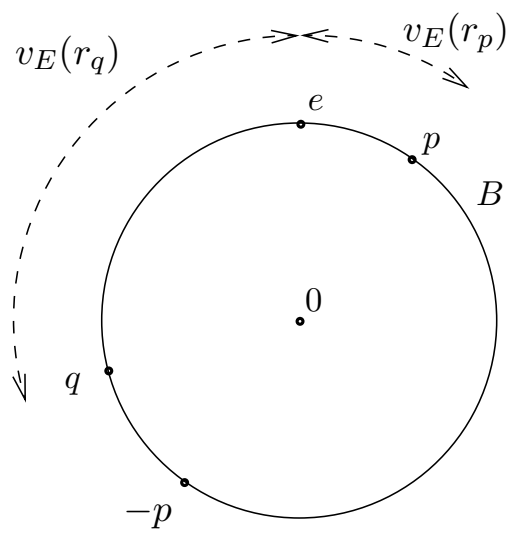

Figure 4. The situation for $p$ and $q$ realizing the diameter of $E_{s c}$.

realize the diameter of $E_{s c}$, by the definition of the rearrangement by spherical caps it is clear that $p, q$ and $e$ belong to a two-dimensional subspace of $\mathbb{R}^{n}$, and by construction

$$
d(e, p) \leq v_{E}\left(r_{p}\right), \quad d(e, q) \leq v_{E}\left(r_{q}\right) .
$$

In particular (see Figure 4), by the triangular inequality

$$
d(-p, q) \geq d(-p, p)-d(p, e)-d(e, q) \geq \pi-\left(v_{E}\left(r_{p}\right)+v_{E}\left(r_{q}\right)\right) .
$$

We can immediately exclude the case when both $v_{E}\left(r_{p}\right)=0$ and $v_{E}\left(r_{q}\right)=0$. Indeed, in this case we have $\operatorname{diam}\left(E_{s c}\right)=\left|r_{p}-r_{q}\right|$, and on the other hand both 
$\partial B_{r_{p}}$ and $\partial B_{r_{q}}$ contain points of $\bar{E}$, which directly implies $\operatorname{diam}(E) \geq\left|r_{p}-r_{q}\right|=$ $\operatorname{diam}\left(E_{s c}\right)$, so the thesis would already be achieved. Therefore, without loss of generality we can assume that

$$
v_{E}\left(r_{q}\right)>0 .
$$

Now consider the set of all the points in $\partial B_{r_{q}}$ that lie at distance larger than $\operatorname{diam}(E)$ from $r_{p} p$

$$
\left\{x \in \partial B_{r_{q}}:\left|r_{p} p-x\right|>\operatorname{diam}(E)\right\} .
$$

If this set is empty, then in particular $\operatorname{diam}\left(E_{s c}\right)=\left|r_{p} p-r_{q} q\right| \leq \operatorname{diam}(E)$; thus we already have the conclusion. Otherwise, the above set is a non-empty spherical cap centered at $-r_{q} p$, and we denote its radius by $r_{q} \psi\left(r_{p}, r_{q}\right)>0$. Thus,

$$
\left\{x \in \partial B_{r_{q}}:\left|r_{p} p-x\right|>\operatorname{diam}(E)\right\}=r_{q} K\left[-p, \psi\left(r_{p}, r_{q}\right)\right] .
$$

Since $r_{p} p$ and $r_{q} q$ realize the diameter of $E_{s c}$, saying that $\operatorname{diam}\left(E_{s c}\right) \leq \operatorname{diam}(E)$ is equivalent to saying that $r_{q} q$ lies at a distance smaller than $\operatorname{diam}(E)$ from $r_{p} p$, which by (3.5) amounts to

$$
d(q,-p) \geq \psi\left(r_{p}, r_{q}\right) .
$$

In turn, in view of (3.3) the thesis is then reduced to check that

$$
v_{E}\left(r_{q}\right)+v_{E}\left(r_{p}\right)+\psi\left(r_{p}, r_{q}\right) \leq \pi .
$$

By the diameter constraint, we have that for every $r_{p} p^{\prime} \in \partial B_{r_{p}} \cap E$,

$$
r_{q} K\left[-p^{\prime}, \psi\left(r_{p}, r_{q}\right)\right] \subset \partial B_{r_{q}} \backslash E .
$$

Therefore

$$
I_{\psi\left(r_{p}, r_{q}\right)} a\left\{\frac{1}{r_{p}}\left(\partial B_{r_{p}} \cap E\right)\right\} \subset \frac{1}{r_{q}}\left\{\partial B_{r_{q}} \backslash E\right\},
$$

where $a: \partial B_{1} \rightarrow \partial B_{1}$ is the antipodal map, i.e., $a(p):=-p$. Thus

$$
\begin{aligned}
\mathcal{H}^{n-1}\left(K\left[p, v_{E}\left(r_{q}\right)\right]\right) & =\frac{\mathcal{H}^{n-1}\left(\partial B_{r_{q}} \cap E\right)}{r_{q}^{n-1}}=\mathcal{H}^{n-1}\left(\partial B_{1}\right)-\frac{\mathcal{H}^{n-1}\left(\partial B_{r_{q}} \backslash E\right)}{r_{q}^{n-1}} \\
& \leq \mathcal{H}^{n-1}\left(\partial B_{1}\right)-\mathcal{H}^{n-1}\left(I_{\psi\left(r_{p}, r_{q}\right)} a\left\{\frac{1}{r_{p}}\left(\partial B_{r_{p}} \cap E\right)\right\}\right) \\
& \leq \mathcal{H}^{n-1}\left(\partial B_{1}\right)-\mathcal{H}^{n-1}\left(I_{\psi\left(r_{p}, r_{q}\right)} K\left[p, v_{E}\left(r_{p}\right)\right]\right),
\end{aligned}
$$

where in the last inequality we have applied (3.2) and the fact that the antipodal map is an isometry. The above inequality (3.7) ensures that $v_{E}\left(r_{p}\right)+\psi\left(r_{p}, r_{q}\right)<\pi$; indeed, otherwise the right term is zero while the left one is strictly positive by (3.4). In turn, since $v_{E}\left(r_{p}\right)+\psi\left(r_{p}, r_{q}\right)<\pi$, then

$$
I_{\psi\left(r_{p}, r_{q}\right)} K\left[p, v_{E}\left(r_{p}\right)\right]=K\left[p, \psi\left(r_{p}, r_{q}\right)+v_{E}\left(r_{p}\right)\right],
$$

which inserted in (3.7) yields

$$
\mathcal{H}^{n-1}\left(K\left[p, v_{E}\left(r_{q}\right)\right]\right) \leq \mathcal{H}^{n-1}\left(K\left[-p, \pi-\left(v_{E}\left(r_{p}\right)+\psi\left(r_{p}, r_{q}\right)\right)\right]\right),
$$

and (3.6) follows by the monotonicity of $\alpha \in(0, \pi) \mapsto \mathcal{H}^{n-1}(K[p, \alpha])$. Thus, we conclude the thesis. 
3.2. Sharp decay rates. We now pass to construct families of nearly optimal sets in the isodiametric inequality showing the optimality of the decay rates in Theorems 1 and 2 ,

Given $\varepsilon \in(0,1), f, g:(0, \varepsilon) \rightarrow(0, \pi)$, and $p \in \partial B_{1}$, let us denote by $E[\varepsilon, f, g, p] \subset$ $\mathbb{R}^{n}$ the set defined by

$$
E[\varepsilon, f, g, p]:=B_{1} \cup \bigcup_{0 \leq t \leq \varepsilon}(1+\varepsilon-t) K[p, f(t)] \backslash \bigcup_{0 \leq t \leq \varepsilon}(1-\varepsilon+t) K[-p, g(t)],
$$

where $K[\cdot, \cdot]$ is the spherical cap defined in (3.1). In the following lemma we provide a sufficient condition for such a set to have diameter equal to 2 , together with an upper bound for its isodiametric deficit. Being interested in nearly optimal sets, we can fix suitable small ranges for the parameters into play. The corresponding explicit constants appearing in the following are not meant to be optimal at all.

Lemma 12. Let $\varepsilon \in\left(0,16^{-2}\right), f:(0, \varepsilon) \rightarrow(0, \pi / 16), g:(0, \varepsilon) \rightarrow(0, \pi / 8)$ be defined as

$$
g(t)=\max \{f(s)+\pi \sqrt{t-s}: 0 \leq s \leq t\}, \quad t \in(0, \varepsilon),
$$

and set $E=E[\varepsilon, f, g, p]$ for some $p \in \partial B_{1}$. Then $\operatorname{diam}(E)=2$, and

$$
\delta(E) \leq \frac{1}{|E|} \int_{0}^{\varepsilon} \mathcal{H}^{n-1}(K[p, g(t)])-\mathcal{H}^{n-1}(K[p, f(t)]) d t
$$

Moreover,

$$
\begin{gathered}
r_{E}^{\text {out }} \geq \frac{\varepsilon}{3}, \\
\inf _{x \in \mathbb{R}^{n}}\left|E \Delta B_{1}(x)\right| \geq \frac{1}{3} \min \left\{c(n) \varepsilon, \int_{\varepsilon / 2}^{\varepsilon} \mathcal{H}^{n-1}(K[p, f(t)]) d t\right\},
\end{gathered}
$$

for some constant $c(n) \in(0, \infty)$ depending only on $n$.

Proof. We divide the proof into several steps.

Step I. Estimate on $\psi(s, t)$. In this first step, we fix any $0 \leq s \leq t \leq \varepsilon \leq 4 / 9$, and we aim to get the estimate (3.12) below for the geodetic radius $\psi(s, t) \in[0, \pi / 2)$ defined by the identity

$$
\partial B_{1-\varepsilon+t} \backslash B_{2}((1+\varepsilon-s) p)=(1-\varepsilon+t) K[-p, \psi(s, t)] .
$$

The problem is essentially two-dimensional, and in suitable coordinates we can set

$$
(1+\varepsilon-s) p=(0,1+\varepsilon-s)
$$

and parameterize the generic point $q \in(1-\varepsilon+t) K[-p, \psi(s, t)]$ as

$$
q=(1-\varepsilon+t)(\sin \varphi,-\cos \varphi), \quad|\varphi|<\psi(s, t) ;
$$

see Figure 5. From the elementary inequality

$$
\cos \varphi \leq 1-\left(\frac{2}{\pi}\right)^{2} \varphi^{2}, \quad \varphi \in\left(-\frac{\pi}{2}, \frac{\pi}{2}\right),
$$




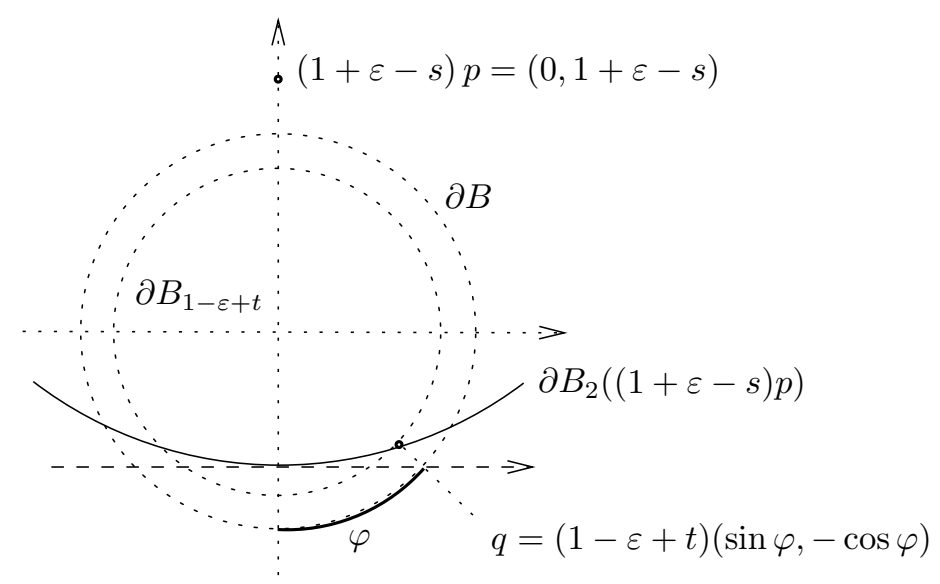

Figure 5. Step one in the proof of Lemma 12

we find that, setting $|\varphi|=\psi(s, t)$,

$$
\begin{aligned}
4 & =|(1+\varepsilon-s) p-q|^{2}=|(-(1-\varepsilon+t) \sin \varphi, 1+\varepsilon-s+(1-\varepsilon+t) \cos \varphi)|^{2} \\
& =(1-\varepsilon+t)^{2}+(1+\varepsilon-s)^{2}+2(1+\varepsilon-s)(1-\varepsilon+t) \cos \varphi \\
& \leq(1-\varepsilon+t)^{2}+(1+\varepsilon-s)^{2}+2(1+\varepsilon-s)(1-\varepsilon+t)\left(1-\left(\frac{2}{\pi}\right)^{2} \varphi^{2}\right) \\
& =(2+t-s)^{2}-\frac{8}{\pi^{2}}(1+\varepsilon-s)(1-\varepsilon+t) \varphi^{2}
\end{aligned}
$$

that is,

$$
\frac{8}{\pi^{2}}(1+\varepsilon-s)(1-\varepsilon+t) \psi(s, t)^{2} \leq(4+t-s)(t-s) .
$$

Taking into account that $1+\varepsilon-s \geq 1$, that $1-\varepsilon+t \geq 5 / 9$, and that $4+t-s \leq 40 / 9$, we conclude that

$$
\psi(s, t) \leq \pi \sqrt{t-s}
$$

Step II. The set $E$ has diameter 2. Given $p, \varepsilon, f, g$ and $E$ as in the statement of the theorem, we now prove that $\operatorname{diam}(E)=2$. The inequality $\operatorname{diam}(E) \geq 2$ is clear, since $\bar{E}$ contains both $(1+\varepsilon) p$ and $(1-\varepsilon)(-p)$. Hence, we concentrate on the opposite inequality.

Then taking two points $q_{0}, q_{1} \in E$, we need to establish that $\left|q_{0}-q_{1}\right| \leq 2$. Since this clearly holds if both points belong to $\bar{B}_{1}$, we assume without loss of generality that $q_{0} \in E \backslash \bar{B}_{1}$, so that $q_{0} \in(1+\varepsilon-s) K[p, f(s)]$ for some $s \in(0, \varepsilon)$. If also $q_{1} \in E \backslash \bar{B}_{1}$, then we have $q_{1} \in(1+\varepsilon-\tilde{s}) K[p, f(\tilde{s})]$ for some $\tilde{s} \in(0, \varepsilon)$, and in particular

$\left|q_{0}-q_{1}\right| \leq\left|q_{0}-(1+\varepsilon-s) p\right|+\left|(1+\varepsilon-\tilde{s}) p-q_{1}\right|+|\tilde{s}-s| \leq f(s)+f(\tilde{s})+\varepsilon \leq 2$,

so we are done. Assuming, instead, that $q_{1} \in E \cap B_{1}$, it is useful to distinguish whether or not $\left|q_{1}\right| \leq 1-\varepsilon+s$. If it is so, then of course we have $\left|q_{0}-q_{1}\right| \leq$ $\left|q_{0}\right|+\left|q_{1}\right| \leq 2$, hence we are again done. Therefore, we are left to consider the last 
possible situation, namely when there exists some $t \in(s, \varepsilon)$ such that

$$
q_{1} \in(1-\varepsilon+t)\left(\partial B_{1} \backslash K[-p, g(t)]\right) .
$$

Notice now that, calling $q_{0}^{\prime}=q_{0} /\left|q_{0}\right|$ and $q_{1}^{\prime}=q_{1} /\left|q_{1}\right|$, we have

$$
d\left(q_{0}^{\prime}, q_{1}^{\prime}\right) \leq d\left(q_{0}^{\prime}, p\right)+d\left(p, q_{1}^{\prime}\right) \leq f(s)+\pi-g(t)<\pi,
$$

where the last inequality comes directly from the definition (3.8) of $g$, because $t>s$ implies

$$
g(t) \geq f(s)+\pi \sqrt{t-s}>f(s) .
$$

As an immediate consequence, we have that $\left|q_{0}-q_{1}\right|$ is maximal if

$$
d\left(q_{0}^{\prime}, q_{1}^{\prime}\right)=\pi-(g(t)-f(s)) .
$$

Therefore, keeping in mind Step I, proving $\left|q_{0}-q_{1}\right| \leq 2$ is equivalent to showing that

$$
g(t)-f(s) \geq \psi(s, t),
$$

which in turn immediately follows by (3.8) and (3.12), since

$$
g(t)-f(s) \geq \pi \sqrt{t-s} \geq \psi(s, t) .
$$

We have then shown that $\operatorname{diam}(E)=2$.

Step III. Proof of (3.9). Since $\operatorname{diam}(E)=2$, we have that

$$
\begin{aligned}
|E| \delta(E) & =\left|B_{1}\right|-|E|=\left|B_{1} \backslash E\right|-\left|E \backslash B_{1}\right| \\
& =\int_{0}^{\varepsilon}(1-\varepsilon+t)^{n-1} \mathcal{H}^{n-1}(K[-p, g(t)])-(1+\varepsilon-t)^{n-1} \mathcal{H}^{n-1}(K[p, f(t)]) d t \\
& \leq \int_{0}^{\varepsilon} \mathcal{H}^{n-1}(K[-p, g(t)])-\mathcal{H}^{n-1}(K[p, f(t)]) d t,
\end{aligned}
$$

that is, (3.9), as required.

Step IV. Proof of (3.10). To show (3.10) we need to prove that, for every $q \in \mathbb{R}^{n}$, one has $r(q) \geq \varepsilon / 3$, where

$$
r(q):=\inf \left\{r>0: E \subset B_{1+r}(q)\right\} .
$$

First of all, if $q \cdot p \geq(3 / 2) \varepsilon$, then since $-(1-\varepsilon) p \in \bar{E}$ we find

$$
1+r(q) \geq|q+(1-\varepsilon) p| \geq q \cdot p+(1-\varepsilon) \geq 1+\frac{\varepsilon}{2},
$$

so (3.10) is true. Similarly, if $q \cdot p \leq \varepsilon / 2$, then by the fact that $(1+\varepsilon) p \in \bar{E}$ we have

$$
1+r(q) \geq|q-(1+\varepsilon) p| \geq 1+\varepsilon-\frac{\varepsilon}{2}=1+\frac{\varepsilon}{2},
$$

and then again (3.10) follows.

Let us finally assume that $\varepsilon / 2<q \cdot p<(3 / 2) \varepsilon$. Since $E$ is axially symmetric, in suitable planar coordinates we may assume that $q=(x, y)$, with $x \leq 0$ and $y \in(\varepsilon / 2,3 \varepsilon / 2)$. Since the point $(\sin g(\varepsilon),-\cos g(\varepsilon))$ belongs to $\bar{E}$, we get

$$
\begin{aligned}
1+r(q) & \geq|(x, y)-(\sin g(\varepsilon),-\cos g(\varepsilon))| \geq|(0, y)-(\sin g(\varepsilon),-\cos g(\varepsilon))| \\
& \geq \sqrt{1+y^{2}+2 y \cos g(\varepsilon)} \geq \sqrt{1+\varepsilon \cos g(\varepsilon)} .
\end{aligned}
$$


Also, since $g<\pi / 8$, we have $\cos g(\varepsilon)>\cos (\pi / 8)>9 / 10$, so that

$$
r(q) \geq \sqrt{1+\frac{9}{10} \varepsilon}-1 \geq \frac{\varepsilon}{3} .
$$

Hence, we have concluded checking (3.10) also in the last case.

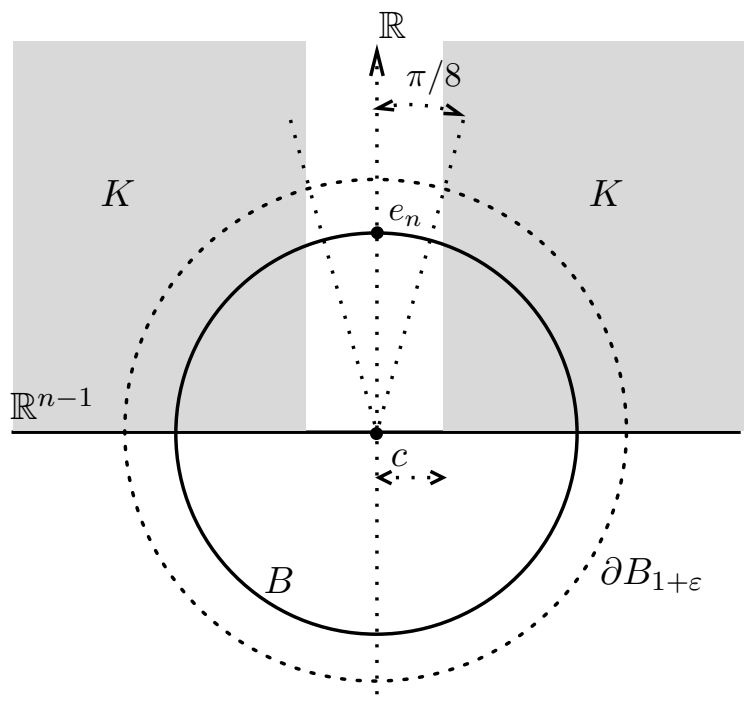

Figure 6 . There exists a constant $c$, depending on $\pi / 8$ and on the fact that $\varepsilon<4 / 9$, such that $E$ agrees with $B_{1}$ on the dark region $K$.

Step V. Proof of (3.11). We are left to the last estimate to show, namely, (3.11). To this end, it is convenient to write $x=\left(x^{\prime}, x_{n}\right) \in \mathbb{R}^{n}=\mathbb{R}^{n-1} \times \mathbb{R}$ and to set $p=e_{n}$. By the same argument used in the proof of [Ma, Lemma 5.2], exploiting the fact that $E$ is axially symmetric with respect to the $e_{n}$-axis, we find that

$$
\inf _{x \in \mathbb{R}^{n}}\left|E \Delta B_{1}(x)\right| \geq \frac{1}{3} \inf _{t \in \mathbb{R}}\left|E \Delta B_{1}\left(t e_{n}\right)\right| .
$$

Since $f<\pi / 8$ on $(0, \varepsilon)$ and $\varepsilon<4 / 9$, there exists a positive constant $c$ such that, if we set

$$
K=\left\{\left(x^{\prime}, x_{n}\right) \in \mathbb{R}^{n}: x_{n}>0,\left|x^{\prime}\right|>c\right\},
$$

then (see Figure 6)

$$
\left(E \Delta B_{1}\right) \cap K=\emptyset .
$$

Evidently, there also exists a positive constant $c(n)$ (roughly speaking, a fraction of the $(n-1)$-dimensional measure of the spherical region $\left.K \cap \partial B_{1}\right)$, such that

$$
\left|\left(B_{1}\left(t e_{n}\right) \Delta B_{1}\right) \cap K\right| \geq 2 c(n) \min \{|t|, 1\}, \quad \forall t \in \mathbb{R} .
$$

Therefore, if $|t| \geq \varepsilon / 2$, then we deduce

$$
\left|E \Delta B_{1}\left(t e_{n}\right)\right| \geq c(n) \varepsilon .
$$

Instead, if $|t|<\varepsilon / 2$, then we surely have

$$
\left\{x \in E:|x|>1+\frac{\varepsilon}{2}\right\} \subset E \Delta B_{1}\left(t e_{n}\right),
$$


so that

$\left|E \Delta B_{1}\left(t e_{n}\right)\right| \geq \int_{\varepsilon / 2}^{\varepsilon}(1+\varepsilon-\tau)^{n-1} \mathcal{H}^{n-1}(K[p, f(\tau)]) d \tau \geq \int_{\varepsilon / 2}^{\varepsilon} \mathcal{H}^{n-1}(K[p, f(\tau)]) d \tau$.

Putting together (3.14) and (3.15), and recalling (3.13), we obtain (3.11).

Example 13 (Optimality of (1.2) and of (1.4) with $n=2$ ). Let $0<\varepsilon<1 / 16^{2}$, and for $t \in(0, \varepsilon)$ consider the functions

$$
\begin{aligned}
f_{\varepsilon}(t) & =\frac{\pi}{16 \varepsilon} t, \\
g_{\varepsilon}(t) & =\max \left\{f_{\varepsilon}(s)+\pi \sqrt{t-s}: 0 \leq s \leq t\right\} .
\end{aligned}
$$

For every $t \in(0, \varepsilon)$ there exists $s(t) \in(0, t)$ such that

$$
\begin{aligned}
0 & \leq g_{\varepsilon}(t)-f_{\varepsilon}(t)=\pi \sqrt{t-s(t)}-\frac{\pi}{16 \varepsilon}(t-s(t)) \\
& \leq \max \left\{\pi \sqrt{\sigma}-\frac{\pi}{16 \varepsilon} \sigma: 0<\sigma<\varepsilon\right\}=4 \pi \varepsilon .
\end{aligned}
$$

Fix $p \in \partial B_{1}$, and consider the family of sets $E_{\varepsilon}=E[\varepsilon, f, g, p]$ defined as in Lemma 12, By construction we have

$$
\int_{\varepsilon / 2}^{\varepsilon} \mathcal{H}^{n-1}(K[p, f(s)]) d s \geq \kappa(n) \varepsilon
$$

for some constant $\kappa(n)$ depending on the dimension $n$ only. Then, by Lemma 12 we have $\operatorname{diam}\left(E_{\varepsilon}\right)=2$,

$$
r_{E_{\varepsilon}}^{\text {out }} \geq \frac{\varepsilon}{3}, \quad \quad \inf _{x \in \mathbb{R}^{n}}\left|E_{\varepsilon} \Delta B_{1}(x)\right| \geq \tilde{\kappa}(n) \varepsilon .
$$

Finally, by (3.9) and (3.16) the following estimate for the isodiametric deficit of the $E_{\varepsilon}$ holds:

$$
\begin{aligned}
|E| \delta\left(E_{\varepsilon}\right) & \leq \int_{0}^{\varepsilon} \mathcal{H}^{n-1}\left(K\left[p, g_{\varepsilon}(t)\right]\right)-\mathcal{H}^{n-1}\left(K\left[p, f_{\varepsilon}(t)\right]\right) d t \\
& \leq C(n) \varepsilon \max _{t \in(0, \varepsilon)}\left|g_{\varepsilon}(t)-f_{\varepsilon}(t)\right| \leq 4 \pi C(n) \varepsilon^{2},
\end{aligned}
$$

with $C(n)$ being a constant depending only on the dimension. Finally, combining this last estimate with (3.17), we derive the optimality of (1.2), as well as of (1.4) for the case $n=2$.

Example 14 (Optimality of (1.4) with $n \geq 4$ ). Let $0<\varepsilon<1 / 16^{2}, 0<\rho<\pi / 16$, and for $t \in(0, \varepsilon)$ consider the functions

$$
\begin{aligned}
& f_{\varepsilon}(t)= \begin{cases}\rho & \text { if } t=0 \\
0 & \text { otherwise }\end{cases} \\
& g_{\varepsilon}(t)=\max \left\{f_{\varepsilon}(s)+\pi \sqrt{t-s}: 0 \leq s \leq t\right\}=\rho+\sqrt{t} .
\end{aligned}
$$

Fix $p \in \partial B_{1}$, and consider the family of sets $E_{\varepsilon}=E[\varepsilon, f, g, p]$ defined as in Lemma 12. Then, by Lemma 12 we have $\operatorname{diam}\left(E_{\varepsilon}\right)=2$, and $r_{E_{\varepsilon}}^{\text {out }} \geq \varepsilon / 3$. By (3.9) 
the following estimate for the isodiametric deficit of $E_{\varepsilon}$ holds:

$$
\begin{aligned}
|E| \delta\left(E_{\varepsilon}\right) & \leq \int_{0}^{\varepsilon} \mathcal{H}^{n-1}\left(K\left[p, g_{\varepsilon}(t)\right]\right)-\mathcal{H}^{n-1}\left(K\left[p, f_{\varepsilon}(t)\right]\right) d t=\int_{0}^{\varepsilon} \mathcal{H}^{n-1}\left(K\left[p, g_{\varepsilon}(t)\right]\right) d t \\
& \leq C(n) \int_{0}^{\varepsilon}(\rho+\sqrt{t})^{n-1} d t \leq C(n) \varepsilon^{(n+1) / 2}+o(\rho),
\end{aligned}
$$

where $o(\rho) \rightarrow 0$ as $\rho \rightarrow 0$. By the arbitrariness of $\rho$ we conclude that (1.4) is sharp also in the case $n \geq 4$.

Example 15 (Optimality of (1.4) with $n=3$ ). Let $n=3$, let $c<\pi / 16$, and for $0<\varepsilon<1 / 16^{2}$ let $\tilde{f}_{\varepsilon}, \tilde{g}_{\varepsilon}:(0, \varepsilon) \mapsto \mathbb{R}$ be defined by

$$
\begin{aligned}
& \tilde{f}_{\varepsilon}(t)=c\left(\frac{t}{\varepsilon}\right)^{|\log \varepsilon|}, \\
& \tilde{g}_{\varepsilon}(t)=\max \left\{\tilde{f}_{\varepsilon}(s)+\pi \sqrt{t-s}: 0 \leq s \leq t\right\} .
\end{aligned}
$$

In order to evaluate $\tilde{g}_{\varepsilon}(t)$, suppose that $0<s(t)<t$ is a critical point for the right hand side in the definition of $\tilde{g}_{\varepsilon}$. Hence, setting for simplicity $\varepsilon=e^{-l}$, i.e., $l=|\log \varepsilon|$, we readily obtain

$$
s(t)^{l-1}=\frac{\pi \varepsilon^{l}}{2 c l \sqrt{t-s(t)}} .
$$

Let $1>\theta>e^{-1 / 2}$ be fixed. We claim that for every $t \geq \theta \varepsilon$, if $\varepsilon$ is small enough (depending on $\theta$ ) there exists a unique positive solution $0<s(t)<t$ of (3.18) satisfying

(i) $\tilde{g}_{\varepsilon}(t)=\tilde{f}_{\varepsilon}(s(t))+\pi \sqrt{t-s(t)}$;

(ii) $\left(\frac{t}{s(t)}\right)^{l} \rightarrow 1$ as $\varepsilon \rightarrow 0$ (or, equivalently, as $l \rightarrow \infty$ );

(iii) $\tilde{f}_{\varepsilon}(t) \geq \tilde{g}_{\varepsilon}(t) / 2$.

In fact, consider the maximization problem which defines $g_{\varepsilon}(t)$. The maximum is surely not attained at $s=t$, because $\sqrt{t-s}$ has a negative infinite slop at $s=t$, while $\tilde{f}_{\varepsilon}$ is regular around $s=t$. To exclude that the maximum is at $s=0$, it is enough to check that $\pi \sqrt{t}<\tilde{f}_{\varepsilon}(t)$. In turn, since the square root is concave while $\tilde{f}_{\varepsilon}$ is convex, we can limit ourselves to checking that $\pi \sqrt{\theta \varepsilon}<\tilde{f}_{\varepsilon}(\theta \varepsilon)$. Finally, this follows by the fact that

$$
\frac{\sqrt{\theta \varepsilon}}{\tilde{f}_{\varepsilon}(\theta \varepsilon)}=c^{-1} \theta^{1 / 2} e^{-l / 2} \theta^{-l}=c^{-1} \theta^{1 / 2}(\sqrt{e} \theta)^{-l} \rightarrow 0,
$$

where the limit is intended for $\varepsilon \rightarrow 0$, or equivalently, for $l \rightarrow \infty$. Summarizing, we have shown that the maximum in the definition of $\tilde{g}_{\varepsilon}(t)$ is attained at some $0<s(t)<t$, for every $t \geq \theta \varepsilon$. Thus, it is clear that $s(t)$ is a solution of (3.18) and that (i) holds.

To show (ii) we emphasize that, for any given $0<\bar{\delta}<1$, we must have

$$
s(\theta \varepsilon) \geq \bar{\delta} \theta \varepsilon \quad \text { for } l \text { large enough. }
$$

Indeed, arguing in a very similar way as in (3.19), it is enough to show that for any $0<\delta \leq \bar{\delta}$ one has $\tilde{f}_{\varepsilon}(\delta \theta \varepsilon)+\pi \sqrt{\theta \varepsilon-\delta \theta \varepsilon}<\tilde{f}_{\varepsilon}(\theta \varepsilon)$, and in turn this easily follows because for $l \rightarrow \infty$ one has

$$
\frac{\tilde{f}_{\varepsilon}(\delta \theta \varepsilon)+\pi \sqrt{(1-\delta) \theta \varepsilon}}{\tilde{f}_{\varepsilon}(\theta \varepsilon)}=\delta^{l}+\pi c^{-1} \sqrt{(1-\delta) \theta} e^{-l / 2} \theta^{-l} \leq \bar{\delta}^{l}+\pi c^{-1}(\sqrt{e} \theta)^{-l} \rightarrow 0 .
$$


Thus, (3.20) is established. Now, since $s(t)$ solves (3.18), we have

$$
t-s(t)=\frac{\pi^{2}}{4 c^{2} l^{2}} s(t)^{2-2 l} \varepsilon^{2 l},
$$

and hence

$$
t=s(t)+\frac{\pi^{2}}{4 c^{2} l^{2}} s(t)^{2-2 l} \varepsilon^{2 l} .
$$

If $\bar{\delta}$ is fixed in such a way that $\bar{\delta} \theta>e^{-1 / 2}$, a quick inspection shows that the function

$$
x \mapsto x+\frac{\pi^{2}}{4 c^{2} l^{2}} x^{2-2 l} \varepsilon^{2 l}
$$

is strictly increasing for $\bar{\delta} \theta \varepsilon<x<\varepsilon$. This observation, together with (3.20), implies at once that, if $\varepsilon$ is small enough, the critical point $s(t)$ is unique and $s(t)$ is increasing on $t \geq \theta \varepsilon$. Moreover, by (3.22) and (3.20) we easily get

$$
1 \leq\left(\frac{t}{s(t)}\right)^{l} \leq\left(1+\left((\bar{\delta} \theta)^{1-2 l} e^{-l}\right)\right)^{l} \leq\left(1+(\bar{\delta} \theta \sqrt{e})^{-2 l}\right)^{l} \rightarrow 1,
$$

so that (ii) follows.

Finally, to prove (iii), notice that it is equivalent to $\tilde{f}_{\varepsilon} /\left(\tilde{g}_{\varepsilon}-\tilde{f}_{\varepsilon}\right) \geq 1$. To prove this last inequality, first notice that, thanks to (3.21) and the fact that $s(t)$ is increasing, $t-s(t)$ is decreasing. Hence, for any $t \geq \theta \varepsilon$ we have, also thanks to (i),

$$
\tilde{g}_{\varepsilon}(t)-\tilde{f}_{\varepsilon}(t)=\tilde{f}_{\varepsilon}(s(t))-\tilde{f}_{\varepsilon}(t)+\pi \sqrt{t-s(t)} \leq \pi \sqrt{t-s(t)} \leq \pi \sqrt{\theta \varepsilon-s(\theta \varepsilon)} .
$$

As a consequence, for $l$ large enough, recalling (ii) and (3.18) we have

$$
\begin{aligned}
\frac{\tilde{f}_{\varepsilon}(t)}{\tilde{g}_{\varepsilon}(t)-\tilde{f}_{\varepsilon}(t)} & \geq \frac{\tilde{f}_{\varepsilon}(\theta \varepsilon)}{\pi \sqrt{\theta \varepsilon-s(\theta \varepsilon)}}=\frac{c \theta^{l}}{\pi \sqrt{\theta \varepsilon-s(\theta \varepsilon)}}=\frac{2 c^{2} l}{\pi^{2}} \theta^{l} \varepsilon^{-l} s(\theta \varepsilon)^{l-1} \\
& =\frac{2 c^{2} l}{\pi^{2}} \theta^{l} \varepsilon^{-l}(\theta \varepsilon)^{l-1}\left(\frac{s(\theta \varepsilon)}{\theta \varepsilon}\right)^{l-1}=\frac{2 c^{2} l}{\theta \pi^{2}} \theta^{2 l} e^{l}\left(\frac{s(\theta \varepsilon)}{\theta \varepsilon}\right)^{l-1} \geq\left(\theta^{2} e\right)^{l} \geq 1,
\end{aligned}
$$

so that (iii) also follows.

We can finally pass to the construction of the nearly optimal set. Let $f_{(1-\theta) \varepsilon}$ : $(0,(1-\theta) \varepsilon) \rightarrow(0, \pi / 16)$ be defined by

$$
f_{(1-\theta) \varepsilon}(t)=\tilde{f}_{\varepsilon}(t+\theta \varepsilon),
$$

and, correspondingly, define $g_{(1-\theta) \varepsilon}:(0,(1-\theta) \varepsilon) \rightarrow(0, \pi / 2)$ as

$$
g_{(1-\theta) \varepsilon}(t)=\max \left\{f_{(1-\theta) \varepsilon}(s)+\pi \sqrt{t-s}: 0 \leq s \leq t\right\} .
$$

Notice that by construction

$$
g_{(1-\theta) \varepsilon}(t) \leq \tilde{g}_{\varepsilon}(t+\theta \varepsilon) \quad \text { for every } t \in[0,(1-\theta) \varepsilon] .
$$

Fix $p \in \partial B_{1}$, and consider the family of sets $E_{(1-\theta) \varepsilon}=E\left[(1-\theta) \varepsilon, f_{(1-\theta) \varepsilon}, g_{(1-\theta) \varepsilon}, p\right]$, defined according to Lemma 12, with $\varepsilon$ replaced by $(1-\theta) \varepsilon$. Then, by Lemma 12 the set $E_{(1-\theta) \varepsilon}$ satisfies the diameter constraint $\operatorname{diam}\left(E_{(1-\theta) \varepsilon}\right)=2$, and

$$
r_{E_{(1-\theta) \varepsilon}^{\text {out }}} \geq \frac{(1-\theta) \varepsilon}{3} .
$$


We are left to estimate the isodiametric deficit of $E_{(1-\theta) \varepsilon}$. In view of (3.9), (3.18) and (3.23), recalling also (ii) and (iii) we have

$$
\begin{aligned}
\left|E_{(1-\theta) \varepsilon}\right| \delta\left(E_{(1-\theta) \varepsilon}\right) & \leq \int_{0}^{(1-\theta) \varepsilon} \mathcal{H}^{2}\left(K\left[p, g_{(1-\theta) \varepsilon}(t)\right]\right)-\mathcal{H}^{2}\left(K\left[p, f_{(1-\theta) \varepsilon}(t)\right]\right) d t \\
& \leq C \int_{\theta \varepsilon}^{\varepsilon}\left(\tilde{g}_{\varepsilon}\right)(t)^{2}-\left(\tilde{f}_{\varepsilon}\right)(t)^{2} d t \leq 3 C \int_{\theta \varepsilon}^{\varepsilon} \tilde{f}_{\varepsilon}(t)\left(\tilde{g}_{\varepsilon}(t)-\tilde{f}_{\varepsilon}(t)\right) d t \\
& \leq 3 C \int_{\theta \varepsilon}^{\varepsilon} c \varepsilon^{-l} t^{l} \pi \sqrt{t-s(t)}=\frac{3 C \pi^{2}}{2 l} \int_{\theta \varepsilon}^{\varepsilon} t^{l} s(t)^{1-l} d t \\
& \leq \frac{C^{\prime}}{l} \int_{\theta \varepsilon}^{\varepsilon} t\left(\frac{t}{s(t)}\right)^{l-1} d t \leq C^{\prime \prime} \frac{\varepsilon^{2}}{|\log \varepsilon|}
\end{aligned}
$$

with $C, C^{\prime}$ and $C^{\prime \prime}$ being three constants. We have thus constructed a family $F_{\varepsilon}=E_{(1-\theta) \varepsilon}$ of sets with $\operatorname{diam}\left(F_{\varepsilon}\right)=2$ and

$$
\limsup _{\varepsilon \rightarrow 0^{+}} \frac{\delta\left(F_{\varepsilon}\right)|\log \varepsilon|}{\varepsilon^{2}}<\infty .
$$

Since by Theorem 2

$$
K_{\text {out }}(3) \sqrt{\delta\left(F_{\varepsilon}\right)\left|\log \delta\left(F_{\varepsilon}\right)\right|} \geq r_{F_{\varepsilon}}^{\text {out }} \geq \frac{(1-\theta) \varepsilon}{3},
$$

we conclude from (3.24) that

$$
\limsup _{\varepsilon \rightarrow 0^{+}} \frac{\sqrt{\delta\left(F_{\varepsilon}\right)\left|\log \delta\left(F_{\varepsilon}\right)\right|}}{r_{F_{\varepsilon}}^{\text {out }}}<\infty,
$$

thus getting the optimality of (1.4) also in the case $n=3$.

\section{ACKNOWLEDGMENT}

The authors thank Andrea Colesanti for his helpful suggestions. This work was supported by the GNAMPA-INDAM through the 2007-2008 research project Disuguaglianze geometrico-funzionali in forma ottimale e quantitativa, by the ERC Advanced Grant 2008 Analytic Techniques for Geometric and Functional Inequalities, and by the ERC Starting Grant n. 258685 AnOptSetCon.

\section{REFERENCES}

[AFN] Angelo Alvino, Vincenzo Ferone, and Carlo Nitsch, A sharp isoperimetric inequality in the plane, J. Eur. Math. Soc. (JEMS) 13 (2011), no. 1, 185-206, DOI 10.4171/JEMS/248. MR2735080(2011k:52007)

[Be] Felix Bernstein, Uber die isoperimetrische Eigenschaft des Kreises auf der Kugeloberfläche und in der Ebene, Math. Ann. 60 (1905), no. 1, 117-136, DOI 10.1007/BF01447496 (German). MR1511289

[Bi] L. Bieberbach, Über eine Extremaleigenschaft des Kreises, Jahresber. Deutsch. Math.Verein. 24 (1915), 247-250.

[Bo] T. Bonnesen, Über das isoperimetrische Defizit ebener Figuren, Math. Ann. 91 (1924), no. 3-4, 252-268, DOI 10.1007/BF01556082 (German). MR.1512192

[Ca] Stefano Campi, Isoperimetric deficit and convex plane sets of maximum translative discrepancy, Geom. Dedicata 43 (1992), no. 1, 71-81, DOI 10.1007/BF00181298. MR1169365 (93d:52012)

[CL1] Marco Cicalese and Gian Paolo Leonardi, A selection principle for the sharp quantitative isoperimetric inequality, Arch. Ration. Mech. Anal. 206 (2012), no. 2, 617-643, DOI 10.1007/s00205-012-0544-1. MR2980529 
[CL2] M. Cicalese, G. P. Leonardi, Best constants for the isoperimetric inequality in quantitative form, preprint.

[EG] Lawrence C. Evans and Ronald F. Gariepy, Measure theory and fine properties of functions, Studies in Advanced Mathematics, CRC Press, Boca Raton, FL, 1992. MR 1158660 (93f:28001)

[FiMP] A. Figalli, F. Maggi, and A. Pratelli, A mass transportation approach to quantitative isoperimetric inequalities, Invent. Math. 182 (2010), no. 1, 167-211, DOI 10.1007/s00222010-0261-z. MR2672283(2011f:49070)

[Fu] Bent Fuglede, Stability in the isoperimetric problem for convex or nearly spherical domains in $\mathbf{R}^{n}$, Trans. Amer. Math. Soc. 314 (1989), no. 2, 619-638, DOI 10.2307/2001401. MR.942426(89m:52016)

[FMP] N. Fusco, F. Maggi, and A. Pratelli, The sharp quantitative isoperimetric inequality, Ann. of Math. (2) 168 (2008), no. 3, 941-980, DOI 10.4007/annals.2008.168.941. MR2456887 (2009k:52021)

[Ga] R. J. Gardner, The Brunn-Minkowski inequality, Bull. Amer. Math. Soc. (N.S.) 39 (2002), no. 3, 355-405, DOI 10.1090/S0273-0979-02-00941-2. MR 1898210(2003f:26035)

[Ho] Ralph Howard, Convex bodies of constant width and constant brightness, Adv. Math. 204 (2006), no. 1, 241-261, DOI 10.1016/j.aim.2005.05.015. MR2233133 (2007f:52004)

[Ma] F. Maggi, Some methods for studying stability in isoperimetric type problems, Bull. Amer. Math. Soc. (N.S.) 45 (2008), no. 3, 367-408, DOI 10.1090/S0273-0979-08-01206-8. MR2402947(2009b:49105)

[Mo] Michael J. Mossinghoff, A \$1 problem, Amer. Math. Monthly 113 (2006), no. 5, 385-402, DOI 10.2307/27641947. MR2225472(2006m:51021)

Department of Mathematica, The University of Texas at Austin, RlM 8.100 2515 Speedway Stop C1200, Austin, Texas 78712-1202

E-mail address: maggi@math.utexas.edu

Dipartmento di Matematics, "G. Castelnuovo", "Sapienza Università di Roma", P.le Aldo Moro 5, I-00185 Roma, Italy

E-mail address: ponsigli@mat.uniroma1.it

Department Mathematik, Universität Erlangen-Nürnberg, Cauerstrasse 11, 91058 Erlangen, Germany

E-mail address: pratelli@math.fau.de 